\title{
Improving Blast Furnace Raceway Blockage Detection. Part 1: Classification of Blockage Events and Processing Framework
}

\author{
Stefan PUTTINGER ${ }^{1 * *}$ and Hugo STOCKER ${ }^{21}$ \\ 1) Department of Particulate Flow Modelling, Johannes Kepler University, Altenbergerstrasse 69, Linz, 4040 Austria. \\ 2) voestalpine Stahl Donawitz GmbH, Kerpelystrasse 199, Leoben, 8700 Austria.
}

(Received on July 26, 2018; accepted on November 9, 2018; J-STAGE Advance published date:

December 19, 2018)

\begin{abstract}
The present paper is the first part of a three paper series discussing raceway blockages in blast furnaces for ironmaking. During raceway blockages the hot blast flow rate can be reduced significantly and in such cases it is beneficial to shut down additional fuel systems like pulverized coal injection (PCI) on that specific tuyere. Currently this is mainly done by a simple threshold comparison of the hot blast flow rate data. Using a constant threshold level cannot account for drifting sensor levels or local changes in burden permeability around the raceway. Thus, the authors investigate various strategies to find more reliable solutions for blockage detection and the shutdown of $\mathrm{PCl}$ branches. Part 1 discusses the different nature of various blockage events on the basis of tuyere camera images and hot blast flow rate data. For the detailed testing of signal processing (part 2) and image processing algorithms (part 3) a universal testbench is presented which also includes a common quality assessment of the results. Based on the collection of tuyere camera images a simple classification of blockage events is established. The analysis of 1500 hours of blast furnace operation data delivers a proper statistics about the frequency of occurrence of blockages on a small size blast furnace. The detailed analysis of hot blast signals and tuyere camera images presented in this paper series aims to improve the understanding of the raceway regions and their influence on optimal blast furnace operation.
\end{abstract}

KEY WORDS: blast furnace; tuyere monitoring; raceway; blockage detection; signal processing.

\section{Introduction}

Blast furnace operation is a trade-off between the opposing targets of constant pig iron quality, and minimal cost per ton of hot metal. The cost of raw materials like iron ore and coal are external factors which cannot be influenced. However, operating the blast furnace at minimal coke rates by replacing some of the coke by additional tuyere fuels reduces the costs and $\mathrm{CO} 2$ emissions per ton of hot metal (thm). These additional fuels can either be of liquid form (e.g. crude oil, tar or natural gas) or of solid form like shredded waste plastics or pulverized coal. Due to constantly low coal prices the PCI technology is more and more replacing other fuel injections. ${ }^{1)}$ However, using high PCI rates also brings some negative effects on blast furnace operation. Sert and Godijn, for example, reported an increased soot content measured in the top gas caused by an incomplete combustion of the injected coal in the raceway. ${ }^{2)}$ Hence, a major issue for optimal coal combustion is to improve the dispersion of coal particles in the raceway and to optimize the conditions for a fast chemical reaction, respectively. These topics have been addressed by many authors over the last decades. While the dispersion of coal particles can

\footnotetext{
* Corresponding author: E-mail: stefan.puttinger@jku.at DOI: https://doi.org/10.2355/isijinternational.ISIJINT-2018-530
}

be influenced by alternative lance tips, ${ }^{3)}$ the use of coaxial lances ${ }^{4,5)}$ or swirl type lances, ${ }^{6}$ ) the combustion can also be improved by using oxy-coal lances. ${ }^{7)}$ A summary of advanced injection technologies can be found in the book of Ishii. $^{8)}$

Operating a blast furnace at maximum PCI rates demands a more sophisticated process control to ensure stable BF operation. Various approaches have been presented in literature for blast furnace process monitoring. As the blast furnace still remains a "black box", most of these approaches use statistical methods to analyze available plant data to identify faulty behavior, ${ }^{9-11)}$ or use machine learning techniques to predict e.g. the permeability index. ${ }^{12)}$ What is common to most of these studies is the fact that they monitor the $\mathrm{BF}$ as a whole. In the case an abnormal behavior is detected, the blast furnace engineers can take operational actions by changing the burden distribution or blast conditions. However, these actions might take effect only after some delay. The last two decades have also brought a huge step in computational capabilities and numerous studies have been conducted to improve the understanding of burden and gas flow inside a BF by applying the discrete element method (DEM), ${ }^{13,14)}$ or coupled models with computational fluid dynamics (CFD-DEM). ${ }^{15-20)}$

Another key factor for better understanding of unstable $\mathrm{BF}$ operation is continuous raceway monitoring. The move- 
ment of coke particles and the behavior of the coal plume in case of a PCI system are good indicators for fault detection. Most literature on raceway monitoring focuses on the coal plume to detect abnormal behavior. ${ }^{6,21-24)}$ Yamahira et al. use the image data of 40 tuyere cameras to detect clogging of the tuyeres caused by backflow of molten iron by statistical methods. ${ }^{25}$ ) Flooding of the tuyeres by molten iron is a major threat on the equipment and human operators. However, the rise of liquid iron level takes place on a rather slow time scale and can be addressed by averaging image data over longer periods. ${ }^{25)}$ In the present study we will concentrate on raceway blockages caused by erratic movements of the burden instead as this phenomenon has not received much attention so far but could be an important step for the better understanding of faulty BF behavior. Such blockages - which shall also be called events in the further discussion - can appear and disappear very quickly and occur on a frequent basis, but the absolute number may vary strongly between different furnaces and operating procedures. Short blockages of only a few seconds duration may be observed several times per hour, longer blockages of more than a minute duration can happen several times a day and can last for about half an hour in extreme cases. While the former kind of events is probably not interesting for blast furnace operation, the continuous injection of coal in the latter case is not desirable and the PCI branch on the affected tuyere should be shut down to avoid accumulation of unburned coal particles.

The goal of blockage detection in the raceway can be tackled by either observing the raceway visually or by checking other plant data like hot blast flow rates for abrupt changes in signal levels. A usual and very simple approach is to compare the hot blast flow rates with a threshold level to shut down PCI branches. As will be discussed later this solution has major shortcomings. Due to the fact that there are numerous possibilities in signal and image processing but almost no literature about processing of BF raceway data, the authors decided to conduct an extensive study to improve raceway blockage detection. However, before testing different signal and image processing algorithms on real $\mathrm{BF}$ data one has to understand the different nature of

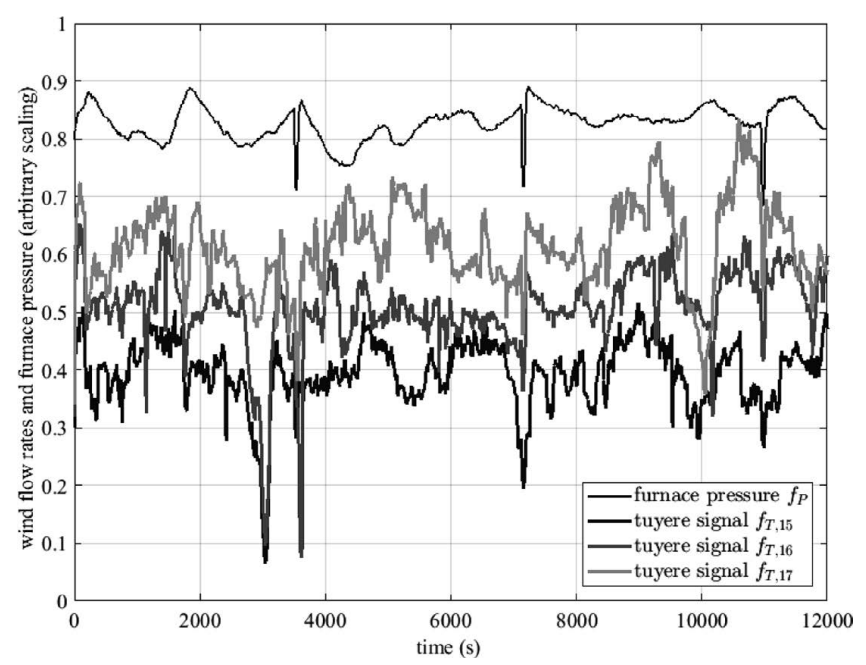

Fig. 1. Examples of tuyere flow rate signals and the furnace pressure signal indicating the switching events of the hot blast stoves. blockage events. Hence, the first part of this paper series discusses various appearances of blockage events and the consequences for signal and image processing, as well as a universal software framework for the testing of different algorithms and a unified quality assessment of the results. Part 2 of this paper series will discuss various signal processing algorithms and their results and part 3 will deal with visual blockage detection via image processing in detail. The authors are aware that every blast furnace is unique in its operating behavior. Although the signals and images presented in this study are from one specific BF, the systematic approach of testing signal and image processing methods on available plant data is universal and can easily be reproduced by the interested reader. As the total length of the content is beyond the limitations of an ordinary journal paper, it was decided to split it into a three-paper series so that all important details can be provided. However, the authors' intention was to keep each part self-contained and brief.

\section{Reasons for Raceway Blockages and their Relation to Blast Flow Rates}

The raceway areas are characterized by a much higher voidage compared to the packed bed in the rest of the blast furnace. High-speed video recordings have shown a highly turbulent motion of coke particles driven by the high inertia of the hot blast. ${ }^{3)}$ Generally speaking, a blast furnace is a counter-current reactor and while the hot blast passes through the porous coke and burden layers upwards, the burden and coke layers themselves move constantly downwards due to the melting of iron ore and the consumption of coke. This movement of the burden excites a force on the raceway which is counter-balanced by the inertia of the hot blast. However, the descent of the burden is not always smooth but can also show unsteady motion of larger areas caused by e.g. wall friction effects or bridge building and collapsing (also known as 'hanging' and 'slips'). ${ }^{26)}$ In the case of such an unsteady movement close to a raceway area the inertia of the hot blast might not be high enough to keep the raceway in its usual extension. This can be visually observed as a complete or partly blocked tuyere. A second reason for blockages is the formation of low porosity zones at the borders of the raceway by unburnt coal particles sticking to the coke. These zones are also termed 'bird's nest'. ${ }^{26,27)}$ Occasionally these shell-type structures can also break and block parts of the raceway for some time. In either case the blast flow rate on the affected tuyere is reduced significantly and the present conditions in the raceway might not be suitable for the combustion of pulverized coal. Figure 1 shows an example of real BF hot blast pressure data for three neighboring tuyeres. One can see significant dips in the signal corresponding to reduced hot blast flow rates. Some of these dips are caused by the changing of the hot blast stoves and correlate with the signal of the absolute blast pressure. However, many other signal dips do not correlate with switching events of the stoves and it can be assumed that they are triggered by abnormal raceway behavior. It is clear from the signals in Fig. 1 that applying a constant threshold level on the blast signals for blockage detection is not an optimal solution, as the average 
signal level can vary significantly between the tuyeres due to inhomogeneous permeability of the burden or signal drift of the pressure sensors due to aging effects.

It can be assumed that a major blockage on one tuyere will also affect the blast flow rates on the neighboring tuyere. However, at the current project state each tuyere is treated individually. In a later project phase it is planned to account for the coupling of neighboring tuyere sections and to implement an additional algorithm which is testing for correlations in the individual tuyere signals.

\section{Discussion of Raceway Conditions and Blockage Events}

\subsection{Collection of Tuyere Images and Blast Data}

To discuss the different nature of blockage events we recorded tuyere images on several arbitrarily chosen tuyeres at BF1 of voestalpine Stahl Donawitz GmbH. In total we have more than 100000 images available for testing purposes. In addition, the hot blast flow rates for the same time periods covered by the image series was stored from the process control system. The examples presented in this paper are taken from one image series consisting of 10000 images recorded at 1 frame per second and thus covering approximately 2 hours and 45 minutes of real blast furnace operation. The corresponding tuyere pressure signal is labeled ' $f_{\mathrm{T}, 15}$ ' in Fig. 1. Note that all analog and digital signals in the figures throughout this paper have been scaled adequately to keep the figures clearly arranged for the reader.

Figure 2 again shows the pressure signal of tuyere 15 where now several regions have been marked and numbered for the following discussion of blockage events. The additional reference signal $f_{R}$ was obtained by manually checking all 10000 images and marking all time intervals with non-ideal raceway conditions (blockages or bad coal plume distribution) for later comparison with the signal and image processing results. Obviously, this manual definition is a subjective approach as different users may interpret the tuyere images differently. However, checking several thousand images gives an overall good impression on the typical raceway conditions of a specific BF and the non-

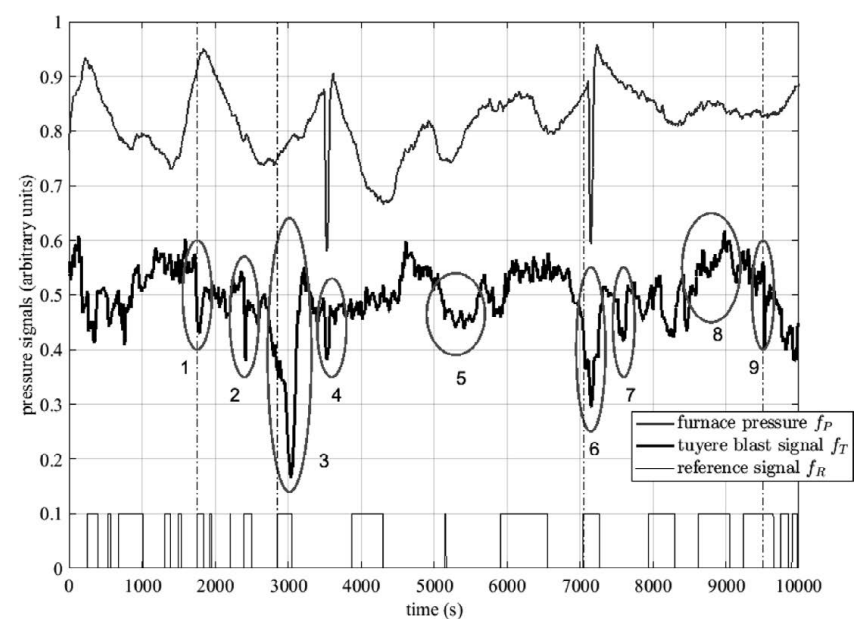

Fig. 2. Test-signal with some blockage events marked and the reference signal obtained by manually checking the tuyere camera images. ideal phases were defined very restrictively on purpose, so the signal and image processing algorithms discussed in part II and part III can be thoroughly tested for their sensitivities. Thus, also very short blockages have been marked in the reference signal as well as phases with diffuse images or unclear raceway condition.

\subsection{Examples of Blockage Events}

For some of the marked events in Fig. 2 sequences of images are presented in Figs. 3 to $\mathbf{6}$ to discuss the temporal evolution of blockage events and different states of the raceway. The timestamps of the individual images are provided as relative times to the first image of the sequence labeled with $t_{x}$ (with $x$ representing the number of the event in Fig. 2).

Figure 3 depicts a rather short tuyere blockage caused by a large lump of agglomerated material. Figure 3(a) shows normal raceway operation. One can see the tip of the PCI lance, part of the coal plume and freely moving coke particles. The bright area in the central left part of the image indicates a void area in the raceway where the high background heat radiation saturates the camera chip to the maximum white level. A few seconds later the blocking structure moves in to the visible area from above (Figs. 3(b) and 3(c)) and finally moves towards the lower right area of the raceway. In Fig. 3(d) the structure then only covers the right part of the raceway area. The left part shows ordinary behavior. The blockage finally disappears after about $30 \mathrm{~s}$.

Figure 4 gives a sequence of images for event \#3 of Fig. 2. In this case some kind of blockage is located further inside the raceway but unlike Fig. 3 there is no large structure with a sharp surface texture visible. There is still room for freely moving coke particles but it is clear that there is a zone of low permeability behind this void region. Checking the consecutive images of this blockage case leads to the conclusion that the blocking material is mainly covered by the impinging coal plume, so instead of seeing a surface texture one sees the coal particles moving around this structure. It is now interesting to compare the situation with the scene in Fig. 3. Although the blockage in Fig. 3(c) seems more dramatic at the first glimpse, there is basically no influence on the particle distribution of the coal plume. As the particles disappear within a certain distance to the lance tip, we can assume that there is still fast combustion of the coal particles. In Fig. 4 the coal particles do not disappear within the same distance to the PCI lance, which leads to the conclusion that the scene in Fig. 4 has a higher impact on the blast flow and the combustion of coal particles. This is indeed the case as event \#3 resulted in a massive reduction of hot blast throughput and was the only event of Fig. 2 where the currently used threshold levels in the process control system led to a shutdown of the PCI branch. Figure 4(d) therefore shows an image where the PCI branch was shut off for about $3 \mathrm{~min}$. The raceway has brightened and cleared again and one can see freely moving coke particles.

Event \#6 which is shown in Fig. 5 is superimposed by a switching event of the hot blast stoves. Thus, at a first glance one could assume that the reduction of blast flow rate seen in Fig. 2 is purely triggered by the switching of the stoves. Indeed, this is not the case and there is already a massive 


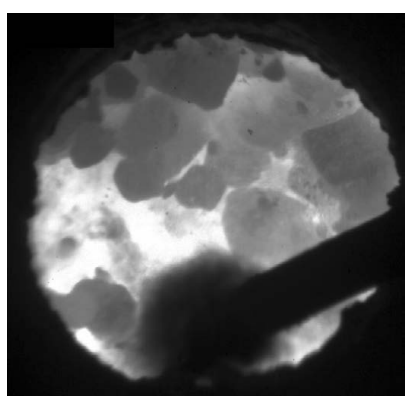

(a) $\mathrm{t}_{1}$

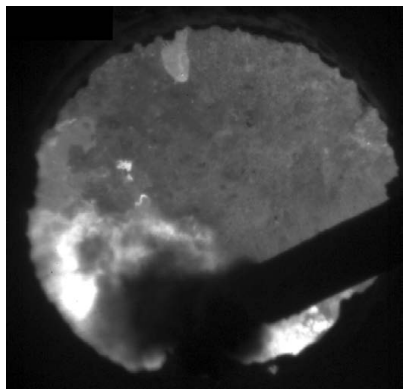

(c) $\mathrm{t}_{1}+6 \mathrm{~s}$

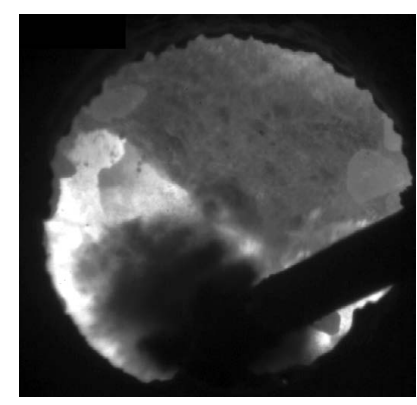

(b) $t_{1}+3 s$

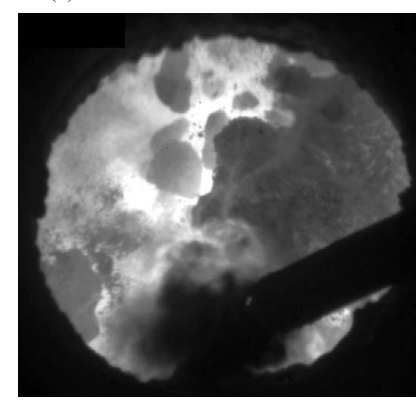

(d) $\mathrm{t}_{1}+20 \mathrm{~s}$

Fig. 3. Images of event $\# 1$ in Fig. 2, (a) normal raceway operation, (b) blocking structure moving in front of the tuyere from above, (c) almost complete blockage of the tuyere, (d) blocking structure moves away from the tuyere towards lower right side.

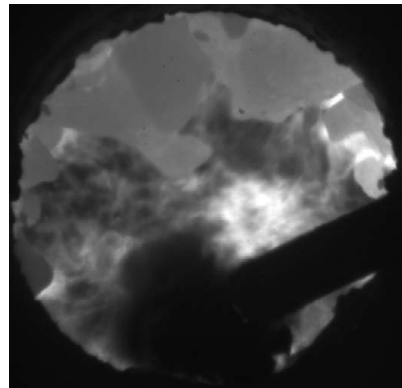

(a) $t_{3}$

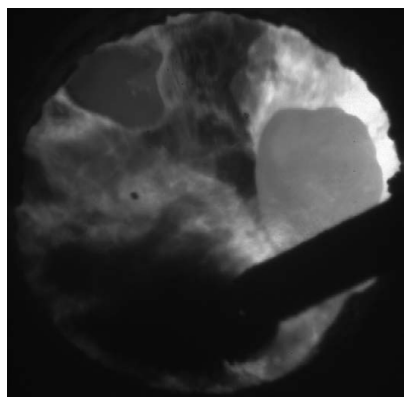

(c) $\mathrm{t}_{3}+50 \mathrm{~s}$

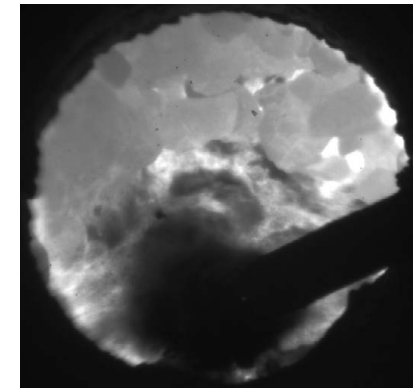

(b) $t_{3}+22 s$

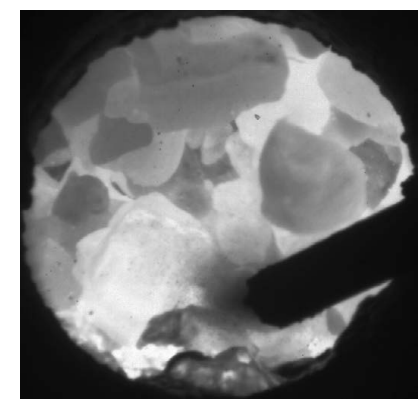

(d) $\mathrm{t}_{3}+114 \mathrm{~s}$

Fig. 4. Images of event $\# 3$ in Fig. 2, (a)-(c) tuyere blockage located further inside the raceway. The low permeability zone does not show clear textures like in Fig. 3, (d) clear raceway after deactivation of the PCI branch.

blockage before the stoves are switched. Thus, filtering of blockage detection results by the stove switching events must be done carefully so that coincidental events are not accidentally erased.

An interesting detail of event \#6 is that while Figs. 5(a) and 5(b) show a blocking structure like in Fig. 3 the visual impression then changes in Fig. 5(c) to the more diffuse

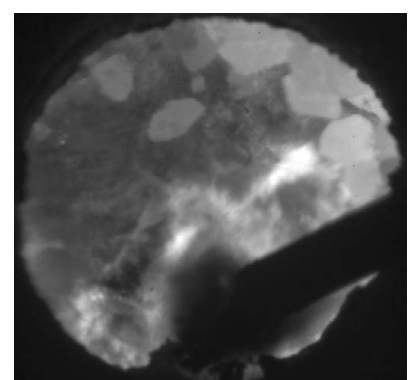

(a) $t_{6}$

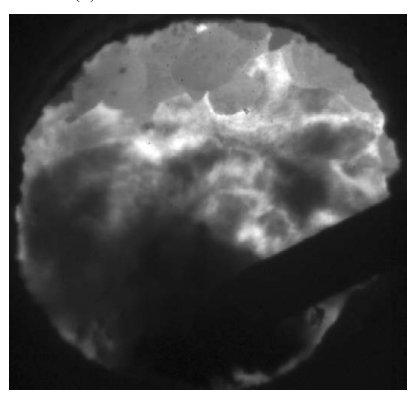

(c) $t_{6}+90 \mathrm{~s}$

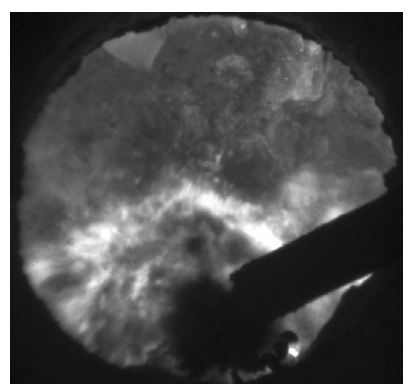

(b) $t_{6}+30 \mathrm{~s}$

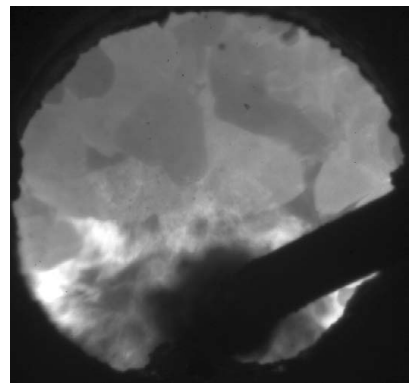

(d) $t_{6}+311 \mathrm{~s}$
Fig. 5. Images of event \#6 in Fig. 2 which is superimposed by the switching of the hot blast stove, (a) and (b) blocking structure with some remaining coke particles in front of the structure, (c) diffuse raceway with unusual coal distribution, (d) return to normal raceway state after more than 5 minutes.

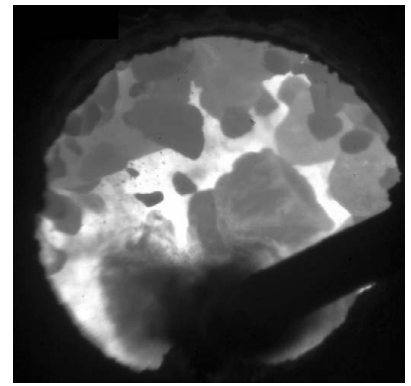

(a) t9

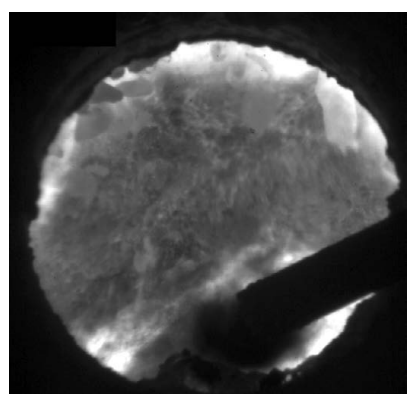

(c) $t_{9}+9 \mathrm{~s}$

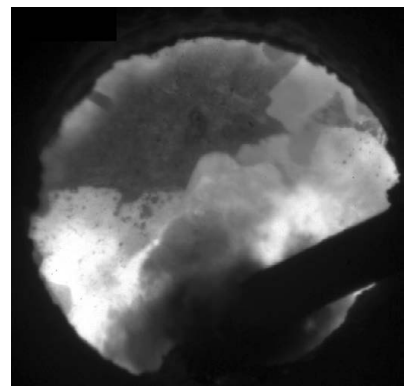

(b) $t_{9}+1 \mathrm{~s}$

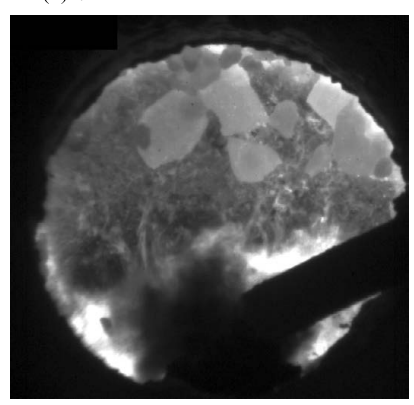

(d) $t_{9}+12 \mathrm{~s}$
Fig. 6. Images of event \#9 in Fig. 2, (a) normal raceway operation, (b) blocking structure moving in front of the tuyere from above, (c) almost complete blockage of the tuyere, (d) blocking structure moves away from the tuyere towards lower right side.

characteristic with abnormal distribution of the coal plume as discussed in event \#3. This could indicate that this abnormal distribution of the coal plume is a typical visual appearance of massive blockages that cause large reductions of the hot blast flow rates. The other way round this would mean that when blocking structures like in Figs. 3 and 6 are visible and still give the impression of a more or less 
sharp surface texture, the conditions for combustion of the injected coal particles are still sufficiently good. Otherwise the visual picture would change to a situation like in Fig. 5(c) where unburnt coal particles cover the major part of the raceway.

Figure 6 shows the evolution of event \#9 and depicts a similar scene as Fig. 3. In Fig. 6(c) it seems that the tuyere is completely blocked, but Fig. 6(d) taken a few seconds later shows that the blockage is located further inside the raceway with a certain distance to the tuyere so there is still room for some coke particles to move around. Therefore, it is not obvious from single tuyere images at which distance to the nozzle a certain structure of agglomerated material appears. It is difficult to measure absolute distances in such harsh conditions with high temperatures and a highly turbulent flow situation. Such measurements have not been conducted within this study, hence, no values can be given on the position of a blockage or the depth of the raceway.

\subsection{Classification of Blockage Events}

Table 1 summarizes and classifies all intervals marked in Fig. 2. Major blockages are relevant for operation and must be detected (events \#3 and \#6). Events which are probably not relevant for $\mathrm{BF}$ operation are classified as minor blockages (events \#1, \#2 and \#9). The signal dip of \#4 is not caused by a blockage but by the changing of the hot blast stove. At the first glance the same reason could be assumed for event \#6 but indeed there is a major blockage which actually starts before the switching of the blast stove as discussed in the previous section. During signal event \#7 no abnormal raceway behavior can be noticed in the tuyere

Table 1. Classification of blockage events marked in Fig. 2.

\begin{tabular}{llllllllll}
\hline Event\# & 1 & 2 & 3 & 4 & 5 & 6 & 7 & 8 & 9 \\
\hline major blockage & & & $\mathrm{X}$ & & & $\mathrm{X}$ & & & \\
minor blockage & $\mathrm{X}$ & $\mathrm{X}$ & & & & & & & $\mathrm{X}$ \\
reduced permeability & & & & & $\mathrm{X}$ & & & $\mathrm{X}$ & \\
no blockage & & & & $\mathrm{X}$ & & & $\mathrm{X}$ & & \\
\hline
\end{tabular}

images, thus not every signal dip does necessarily correlate with a blockage event. In the intervals marked as \#5 and \#8 the raceway shows a rather diffuse and darker appearance but no complete blockage is visible. It can be assumed that the surrounding zone of the raceway has a reduced permeability, which comes along with longer residence time of coal particles.

Although not all blockages marked in the reference signal are relevant for blast furnace operation, the event detection algorithms should be as sensitive as possible in the test phase. For a later online implementation of the blockage detection in the BF process control system, the sensitivities of the algorithms can be reduced as needed according to the frequency and significance of the events on a specific tuyere. However, only if most of the suspicious raceway conditions are detected correctly, one can build a sound statistics of blockage events which can later be used to find correlations with other data from blast furnace operation (e.g. burden charging, coke quality etc.).

\section{Unified Software Testbench and Quality Assessment}

To test different signal and image processing algorithms for their abilities to detect raceway blockages a universal software framework and common quality assessment is needed to compare the results and obtain objective measures. As these parts are the common basis for the discussion of signal and image processing results in part 2 and part 3 of this paper, we give a detailed description of the software testbench here. To demonstrate the processing procedure a dummy algorithm has been implemented which simply reproduces the threshold comparison with the tuyere blast signals as it is currently implemented in the process control system. The results of this dummy algorithm provide the long-term statistics discussed in section 5 .

\subsection{Data Processing Framework}

For efficient testing of the algorithms a modular software test-bench was set up and implemented in Matlab (Fig. 7). The actual processing algorithms are implemented as plugin-functions, so that the test-bench can easily be extended with new features. As Matlab is based on an interpreter,

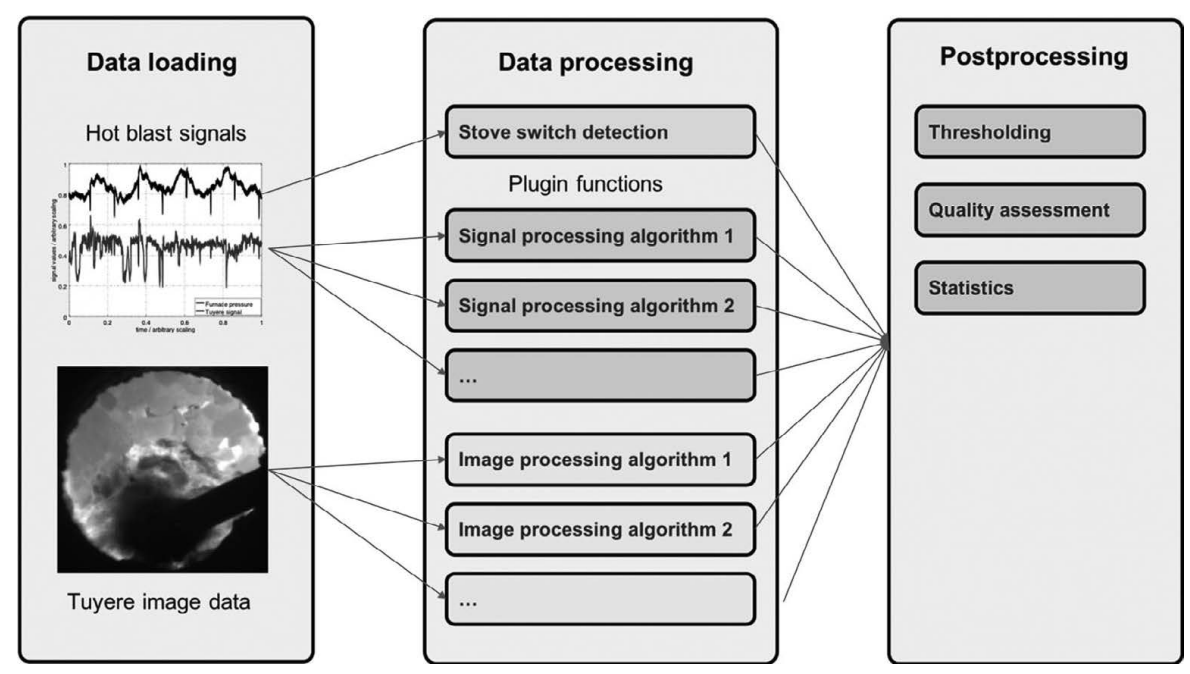

Fig. 7. Outline of the software testbench for the processing of blast signals and tuyere images. 
execution times are obviously longer than with compiled code written in $\mathrm{C}$ or $\mathrm{C}++$, but for prototyping purposes this is not important and the computational efficiency of various algorithms can still be evaluated on a relative scale.

A final implementation of a blockage detection algorithm in the process control system needs to provide a digital yes/ no signal (to switch off PCI branches). Thus, the analog results $f_{S}$ of the tested algorithms are finally converted to digital blockage signals $f_{B}$ which can then be used for direct comparison with the reference signal $f_{R}$. To keep this last conversion as universal as possible all analog result signals $f_{S}$ are normalized to the range $0 . .1$. The conversion of $f_{S}$ to the digital $0 / 1$ blockage signal $f_{B}$ is then based on two threshold levels. If $f_{S}<t h_{o n}$ the blockage signal is 1 , if $f_{S}>t h_{\text {off }}$ the signal will be off again, respectively. As $f_{S}$ is normalized, the threshold levels $t h_{o n}$ and $t h_{\text {off }}$ must also be in the range $0 . .1$.

During the time intervals of hot blast stove switching the signal processing algorithms are deactivated for $120 \mathrm{~s}$ and $f_{S}=f_{B}=0$. In the result plots the state of the hot blast stoves is indicated with a separate digital signal $f_{C}$ to indicate these intervals. The switching events of the hot blast stoves itself is extracted from the furnace pressure signal $f_{P}$, but as these events come along with a sharp negative peak, they are easy to detect and the procedure is not discussed here in detail.

\subsection{Systematic Quantitative Assessment of the Results}

The test-bench can run any set of hot blast data or series of tuyere camera data of any length. To obtain comparable quality measures on the number of found events two different ways of result assessment have been implemented. Obviously, the assessment procedures require a reference signal $f_{R}$ which needs to be generated by manually checking the saved sets of tuyere images. As this is a very time-consuming task the quality assessment can only be performed for rather short time intervals like the set of test-data discussed in section 3.2.

Version 1 of the quality assessment calculates the time stamps with matching decision, missing state or false positive results. This can be done by simple binary operators according to

$$
\begin{aligned}
& r_{m a}=f_{B} \wedge f_{R}, \\
& r_{m i}=f_{B} \vee f_{R}, \\
& r_{f p}=f_{B} \wedge \neg f_{R},
\end{aligned}
$$

where $r_{m a}$ denotes the signal of matching timestamps, $r_{m i}$ the signal of missing timestamps and $r_{f p}$ the signal of false positive timestamps, respectively. An example is plotted in Fig. 8.

The signals $r_{m a}, r_{m i}$ and $r_{f p}$ can be further used to calculate quality measures by summing all ' 1 ' timestamps. This will result in three scalar values giving the accumulated time where the calculated signal and the reference signal match, as well as the total missed and false positive signal time, respectively. The values will be given in relative numbers with respect to the sum of ' 1 '-states of the reference signal $f_{R}$ for the 'match' and 'missed' signals, and the total time of the reference signal for the 'false positive' signal as outlined

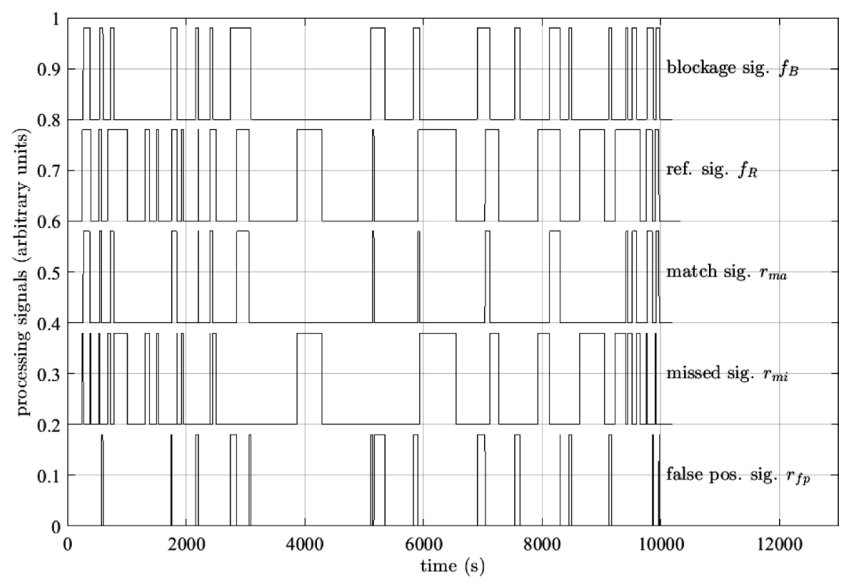

Fig. 8. Version 1 of result quality assessment. $f_{B}$ is the calculated blockage signal and $f_{R}$ is the manually defined reference signal. The signals $r_{m a}, r_{m i}$ and $r_{f p}$ represent the matching, missing and false positive time stamps as defined by Eqs. (1), (2) and (3), respectively.

in Eqs. (4), (5) and (6).

$$
\begin{gathered}
t_{s, m a}=\frac{\sum_{k=1}^{N} r_{m a}(k)}{\sum_{k=1}^{N} f_{R}(k)} . \\
t_{s, m i}=\frac{\sum_{k=1}^{N} r_{m i}(k)}{\sum_{k=1}^{N} f_{R}(k)} . \\
t_{s, f p}=\frac{1}{N} \sum_{k=1}^{N} r_{f p}(k) .
\end{gathered}
$$

This kind of assessment is very strict and might give a too negative view on the results, for example in cases where an algorithm indeed can detect a blockage but does not deliver the duration correctly. In such cases any timestamp with a differing state between the calculated signal $f_{B}$ and the reference signal $f_{R}$ contributes to the cumulative number of missed and false positive event time $t_{s, m i}$ and $t_{s, f p}$, respectively. As an example, the event around $t=7000$ $\mathrm{s}$ in Fig. 8 is detected earlier but also finishes earlier in $f_{B}$ than in $f_{R}$ and thus contributes to $t_{s, m a}, t_{s, m i}$ and $t_{s, f p}$, although one could argue that it was detected correctly if the starting point and duration of a blockage event are not considered as the most important factors in real BF operation. For a less strict assessment it would therefore be sufficient if a signal processing algorithm detects at least one blockage within a time period marked as a blockage in $f_{R}$. Thus we simply want to count the number of detected events, as well as the missed and false events. To achieve this, version 2 of the signal assessment is based on a state-machine and uses only the timestamps of state transitions. A blockage event is considered as detected regardless of the time offset relative to the positive ramp of the reference signal. Hence, the calculated blockage signal can detect the blockage earlier or later than the reference signal. As long as there is a temporal overlap of both signals, the event is marked as correctly detected (Fig. 9). This is a less rigorous assessment of the results than version 1 but also neglects all time delays in the blockage detection. To quantify the time offset 


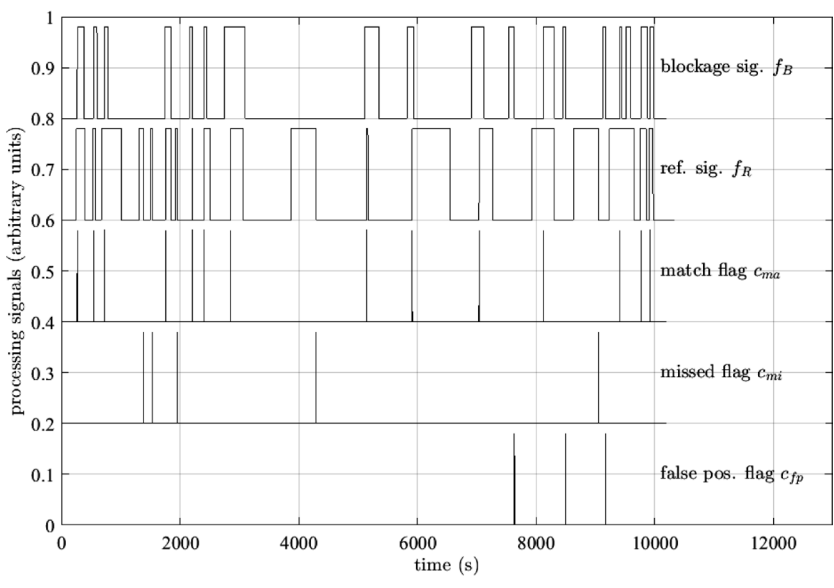

Fig. 9. Version 2 of result quality assessment. $f_{B}$ is the calculated blockage signal and $f_{R}$ is the manually defined reference signal. The signals $c_{m a}, c_{m i}$ and $c_{f p}$ represent the counter signals for matching, missing and false positive events, respectively.

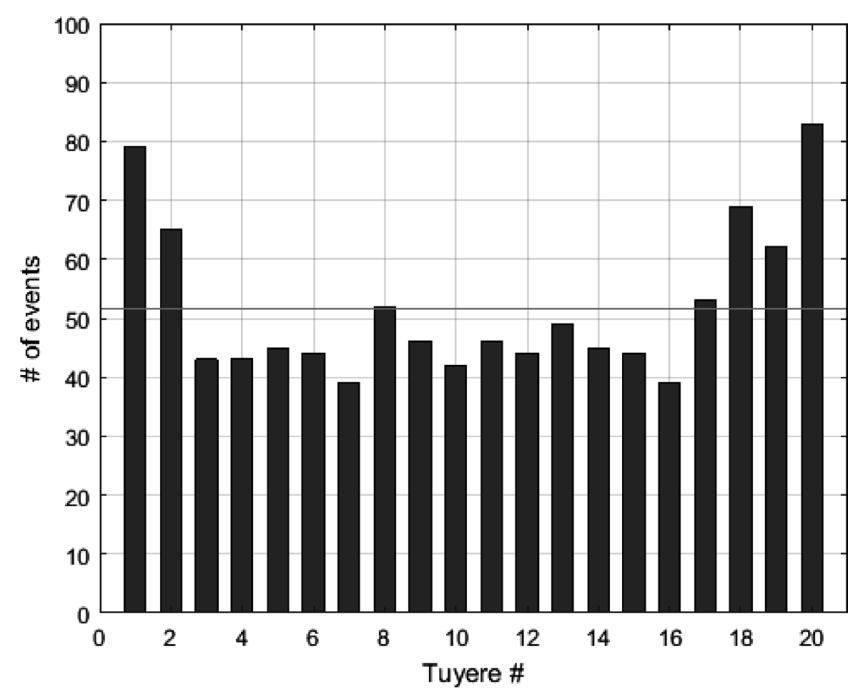

Fig. 10. Number of PCI branch shutdown events on each of the 20 tuyeres of BF1 at voestalpine Donawitz for a period of 1500 operation hours from January to March 2018. The horizontal line marks the averaged value of all tuyeres.

between the state-changes in $f_{R}$ and $f_{B}$ by one scalar value we accumulated the offsets for all events to an overall time delay number $t_{D}$. To obtain minimal time delays in blockage detection, $t_{D}$ should be as small as possible.

\section{Long Term Statistics of Raceway Blockages}

To obtain a valid statistics of blockage events beyond the rather short sets of test-data, we saved 1500 hours of hot blast tuyere signals from the process control system covering a time span from January to March 2018. The currently implemented primitive thresholding of the tuyere signals was implemented as a dummy algorithm named 'A0' (to be consistent with the nomenclature used in part 2 and part 3 ). The plugin function actually does nothing except applying an 'on' threshold of $t h_{o n}=0.05$ for all tuyeres except \#3, \#5 and \#20 where the threshold is set to $3 \%$. In our test system the 'off' threshold was set to $t h_{\text {off }}=0.5$. In fact an off threshold is not present in the current process automation system and the operators have to manually switch back on the PCI branches in case of deactivation. A0 thus will deliver the number of blockages where the process control system indeed has shut down a PCI branch.

Figure 10 gives the number of shutdown events per tuyere for the investigated time span. The statistics show and increased number of shutdown events on the neighboring tuyeres 18,19,20,1 and 2. The overall average of shutdown events results to 0.82 events per tuyere per day.

\section{Summary \& Outlook}

Running a blast furnace at high PCI rates demands improved process monitoring and control to ensure stable operation. The reliable shutdown of PCI branches during operating conditions where an efficient combustion of the injected coal is not possible is one factor to reduce possibly negative effects of high coal rates on blast furnace operation. This inherently brings the need for improved tuyere monitoring to assess the current state of the raceway and detect blockages at an early state. This first part of a threepaper series gives a deeper insight into various appearances of raceway blockages and discusses the visual impression of such events and their influence hot blast flow rates. The obtained sets of test data allow a basic classification of blockage events. In addition, the analysis of 1500 hours of BF operation delivered realistic numbers of the occurrence of tuyere blockages.

To provide a common basis for the testing of signal and image processing algorithms we present a testing framework and common quality assessment of the results. This framework will be used in part 2 which will present the results of different signal processing methods to improve the currently used solution of a primitive threshold comparison of the hot blast flow rates, as well as in part 3 where we will discuss various approaches for visual blockage detection based on tuyere camera images. Having a proper solution for both processing strategies, image processing as well as signal processing of blast data, provides a good basis for evaluating combined approaches for an optimal tuyere blockage detection system and a better understanding of abnormal raceway conditions.

\section{Acknowledgements}

The authors gratefully acknowledge the funding support of K1-MET GmbH, metallurgical competence center. The research program of the competence center K1-MET is supported by COMET, the Austrian program for competence centers.

\section{Nomenclature}

$c_{m a}$ : counter signal for matched events

$c_{m i}$ : counter signal for missed events

$c_{f p}$ : counter signal for false positive events

$f_{B}$ : digital blockage signal derived from $f_{S}$

$f_{C}$ : digital signal indicating hot blast stove switching events

$f_{P}$ : furnace pressure signal

$f_{R}$ : manually defined reference blockage signal

$f_{S}$ : analog result signals of the processing algorithms 
$f_{T}$ : tuyere hot blast signal

$k$ : time index of discrete signals

$r_{m a}$ : result signal marking all matching time indices in signals in $f_{M}$ and $f_{C}$

$r_{m i}$ : result signal marking all missing time indices in signal $f_{C}$

$r_{f p}$ : result signal marking all false positive time indices in signal $f_{C}$

$t_{D}$ : sum of time delays in the matched events of $f_{B}$ and $f_{R}$

$t_{s, m a}$ : sum of time indices with matching signal in $f_{M}$ and $f_{C}$

$t_{s, m i}$ : sum of time indices with missing signal in $f_{C}$

$t_{s, f p}$ : sum of time indices with false positive signal in $f_{c}$

$t h_{o n}$ : 'on' threshold level, $f_{B}$ is set to 1 when $f_{S}<t h_{o n}$

thoff: 'off' threshold level, $f_{B}$ is set to 0 when $f_{S}>$ th off

\section{REFERENCES}

1) A. M. Carpenter: Use of PCI in Blast Furnaces, IEA Clean Coal Centre, London, (2006), 5.

2) D. Sert and R. Godijn: Assessment of Industrial Operation at Low Coke Rate and Coal Injection in Excess of 200kg/Thm, EU Report 20107, European Comission, Brussels, (2002), 79.

3) S. Puttinger, S. Pirker, H. Stocker and E. Schuster: 5th Int. Conf. on Modelling and Simulation of Metallurgical Processes in Steelmaking (STEELSIM 2013), Czech Metallurgical Society, Ostrava, (2013), T1-4.

4) H. Liu, W. Cao, J. Xu, W. Li, X. Guo and Z. Sun: Powder Technol., 225 (2012), 206.

5) C. H. Birzer, P. A. M. Kalt and G. J. Nathan: Int. J. Multiph. Flow, 41 (2012), 13.

6) P. R. Austin, S. J. Chew, D. Maldonado, J. G. Mathieson, D. J. Pinson, H. Rogers, M. Walsh, B. D. Wright and P. Zulli: 6th European Coke and Ironmaking Cong., Stahl-Institut VDEh, Düsseldorf, (2011),
FN1

7) S. W. Du, C. P. Yeh, W. H. Chen, C. H. Tsai and J. A. Lucas: Fuel, 143 (2015), 98.

8) Advanced Pulverized Coal Injection Technology and Blast Furnace Operation, ed. by K. Ishii, Pergamon Press, Oxford, (2000), 123.

9) T. Zhang, H. Ye, W. Wang and H. Zhang: ISIJ Int., 54 (2014), 2334.

10) H. Tian and A. Wang: ISIJ Int., 50 (2010), 738.

11) B. Zhou, H. Ye, H. Zhang and M. Li: Control Eng. Pract., 47 (2016),

12) X. Su, S. Zhang, Y. Yin and W. Xiao: J. Franklin Inst., 355 (2018), 1663.

13) M. Fukuda, J. Suzuki, H. Kawai and H. Nogami: ISIJ Int., 55 (2015), 1291.

14) Z. G. Luo, H. Zhou, T. Zhang, Y. You, L. J. Zhang, Z. S. Zou and Y. S. Shen: Powder Technol., 314 (2017), 102.

15) A. T. Adema, Y. Yang and R. Boom: ISIJ Int., 50 (2010), 954.

16) S. Yuu, T. Umekage, S. Matsuzaki, M. Kadowaki and K. Kunitomo: ISIJ Int., 50 (2010), 962.

17) S. Natsui, H. Nogami, S. Ueda, J. Kano, R. Inoue and T. Ariyama: ISIJ Int., 51 (2011), 41.

18) H. Kurosawa, S. Matsuhashi, S. Natsui, T. Kon, S. Ueda, R. Inoue and T. Ariyama: ISIJ Int., 52 (2012), 1010.

19) F. Bambauer, S. Wirtz, V. Scherer and H. Bartusch: Powder Technol., 334 (2018), 53.

20) T. Lichtenegger and S. Pirker: Powder Technol., 325 (2018), 698.

21) J. Daoud and I. Nipl: Master thesis, Lulea University of Technology, (2000), http://ltu.diva-portal.org/smash/record.jsf?pid=diva2\%3A102 9982\&dswid $=-7053$, (accessed 2017-05-13).

22) W. Birk, O. Marklund and A. Medvedev: IEEE Trans. Ind. Appl., 38 (2002), 571.

23) Y. Wang, Y. Hu, G. Yang, T. Qin and W. Yuan: Procedia Eng., 102 (2015), 265.

24) R. Zhang, S. Cheng and C. Guo: ISIJ Int., 58 (2018), 244.

25) N. Yamahira, T. Hirata, K. Tsuda, Y. Morikawa and Y. Takata: Proc. SPIE, Vol. 9971, The International Society for Optics and Photonics, Bellingham, (2016), 997113-1.

26) M. Geerdes, R. Chaigneau, I. Kurunov, O. Lingiardi and J. Ricketts: Modern Blast Furnace Inronmaking, IOS Press, Amsterdam, (2015), 66.

27) P. Cavaliere: Ironmaking and Steelmaking Processes, Springer, Berlin, (2016), 173. 